\title{
ON THE MODELING OF THIN-WALLED MEMBER ASSEMBLIES COMBINING SHELL AND GBT-BASED BEAM FINITE ELEMENTS: THE LINEAR AND BIFURCATION CASE
}

\author{
DAVID MANTA ${ }^{1}$, RODRIGO GONÇALVES ${ }^{2}$ AND DINAR CAMOTIM ${ }^{3}$ \\ ${ }^{1}$ CERIS and Departamento de Engenharia Civil, Faculdade de Ciências e Tecnologia, Universidade \\ Nova de Lisboa, 2829-516, Caparica, Portugal. E-mail: d.manta@campus.fct.unl.pt \\ ${ }^{2}$ CERIS and Departamento de Engenharia Civil, Faculdade de Ciências e Tecnologia, Universidade \\ Nova de Lisboa, 2829-516, Caparica, Portugal. E-mail: rodrigo.goncalves@ffct.unl \\ ${ }^{3}$ CERIS, ICIST, DECivil, Instituto Superior Técnico, Universidade de Lisboa, Av. Rovisco Pais, \\ 1049-001 Lisboa, Portugal. E-mail: dcamotim@civil.ist.utl.pt
}

Key words: Generalised Beam Theory (GBT), Shell finite elements, cross-section deformation, local-distortional-global buckling.

\begin{abstract}
In this paper, a general and efficient approach to model thin-walled members and frames with complex geometries (including tapered segments and holes). The approach combines shell and GBT-based (beam) finite elements, using each of them where it is most efficient: (i) shell elements in the plastic and geometrically complex zones, and (ii) GBT elements in the prismatic and elastic zones. To illustrate the capabilities and potential of the proposed approach, a set of numerical examples are presented, concerning linear, bifurcation (linear stability) and first-order plastic zone analyses. The examples analysed involve (i) members with tapered segments, (ii) members with holes and (iii) tapered beam-column assemblies. For validation and comparison purposes, full shell finite element solutions are provided and it is demonstrated that the proposed approach yields very accurate solutions in all cases, while involving much less DOFs.
\end{abstract}

\section{INTRODUCTION}

Generalised Beam Theory (GBT) is nowadays a well-established thin-walled bar theory that efficiently accounts for cross-section in-plane and/or out-of-plane (warping) deformation. The efficiency of GBT stems from its modal decomposition features, i.e., the fact that the member deformed configuration is expressed as a linear combination of so-called "cross-section deformation modes", which have a clear and hierarchical physical/structural nature. The advantages of this theory are particularly evident in linear and bifurcation (linear stability) analyses, where the adoption of just a small set of deformation modes already provides very accurate solutions. Moreover, semi-analytical or even analytical solutions are often achieved, providing significant insight into the mechanics of the problem under consideration. The foundations of GBT were set by Schardt [1,2] (see http://vtb.info for related bibliography), but recently several researchers have made significant contributions, particularly the Lisbon-based 
research group (see http://www.civil.ist.utl.pt/gbt for a list of publications).

Although GBT was developed for prismatic bars, efforts have been undertaken to extend its scope to moderately tapered members [3], conical shells [4,5] and members with holes [6], the latter at the expense of using a refined cross-section discretisation and taking into account only the membrane longitudinal normal pre-buckling stresses. A more general approach to handle members with holes, discrete thickness variations and physical/geometrical non-linearities was proposed in [7], using non-orthogonal deformation modes to enable modelling complex geometries and enhancing the computational cost (the participations of the orthogonal modes are retrieved by post-processing). A linear formulation for bars with circular axis was proposed in $[8,9]$, while frames were dealt with in [10-13], using carefully chosen nodal constraints.

The application of GBT to non-linear materials is most effective in plastic bifurcation (linear stability) analyses $[14,15]$. For calculating non-linear equilibrium paths, even though the GBT modal features are still enlightening, the computational gains are partially lost due to the fact that (i) a large number of deformation modes must be included in the analysis, leading to DOF numbers of the same magnitude of those in similarly accurate shell element models, and (ii) large and non-sparse element stiffness matrices are obtained. Providing a remedy for these disadvantages was the aim of several strategies developed for steel plasticity and concrete cracking/crushing: the use of (i) stress constraints that enable reducing the number of deformation modes [16-19] and (ii) shell-like stress resultant yield functions to reduce the modes and avoid through-thickness numerical integration, which has a high computational cost [16,20]. As stated before, even though the approach proposed in [7] can handle
physical/materially non-linearitics quite efficiently, it still lacks the generality of a pure shell
finite element model.
The present paper proposes an alternative, general and efficient approach, combining shell
and GBT-based (beann) finite elements, each one employed where it is most effective: (i) shell
elements in the plastic and/or geometrically complex zones (tanered parts, holes, connections, etc.), and (ii) GBT elements in the prismatic and elastic zones. Following this approach, one

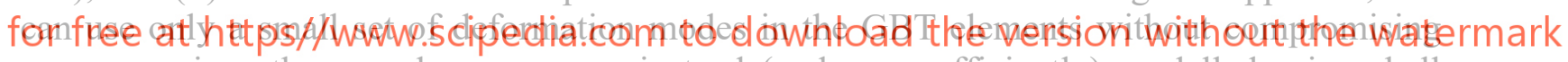
accuracy, since the complex zones are instead (and more efficiently) modelled using shell elements. The capabilities and potential of the proposed approach are illustrated and discussed by examining several numerical examples, involving linear (first-order), bifurcation (linear stability) and first-order plastic zone analyses. These examples deal with (i) members with tapered segments, (ii) members with holes and (iii) frames with complex beam-column assemblies. For validation and comparison purposes, full shell finite element solutions are also shown, and in all cases an excellent match is observed.

Concerning the notation, scalars are represented by italic letters and vectors/matrices by bold letters. Identity matrices are displayed as $\mathbf{1}$ and null matrices or vectors as $\mathbf{0}$. The conventional Euclidean inner product between two vectors of arbitrary dimension $\boldsymbol{a}$ and $\boldsymbol{b}$ is written as $\boldsymbol{a} \cdot \boldsymbol{b}$, a derivative is represented by a subscript comma (e.g., $f_{, a}=\partial f / \partial a$ ). Moreover, a virtual variation is denoted by $\delta$ and an incremental/iterative variation by $\Delta$. Finally, $h$ is the shell/wall thickness and the membrane/bending/through-thickness shear terms are identified by indices $M, B$ and $S$, respectively. 


\section{ADOPTED SHELL AND GBT-BASED FINITE ELEMENTS}

The equilibrium equations and their linearization are first presented. The virtual work statement is written in terms of the work-conjugated pair $\boldsymbol{S}-\boldsymbol{E}$, second Piola-Kirchhoff stresses and Green-Lagrange strains, in a Voigt-like notation, i.e.,

$$
\delta W=-\int_{V} \delta \boldsymbol{E} \cdot \boldsymbol{S} \mathrm{d} V+\int_{\Omega} \delta \overline{\boldsymbol{U}} \cdot \boldsymbol{q} \mathrm{d} \Omega=0,
$$

where $V$ and $\Omega$ are the thin-walled member volume and mid-surface, respectively, at the initial configuration, $\boldsymbol{q}$ are the external loads (for simplicity, applied only at the element mid-surface) and $\boldsymbol{U}$ is the displacement vector ( $\overline{\boldsymbol{U}}$ is its mid-surface component). For a linear elastic material, $\boldsymbol{S}=\boldsymbol{C} \boldsymbol{E}$, where $\boldsymbol{C}$ is the constitutive matrix. For elastoplastic materials, the incremental form of the constitutive equations is given by

$$
\mathrm{d} S=C_{c t} \mathrm{~d} E,
$$

where $C_{c t}$ is the tangent elastoplastic constitutive matrix consistent with the stress update algorithm employed - the backward Euler return algorithm [21]. For the non-linear case, the linearization of Eq. (1) is required to obtain the element tangent stiffness matrix and nodal force vector, leading to

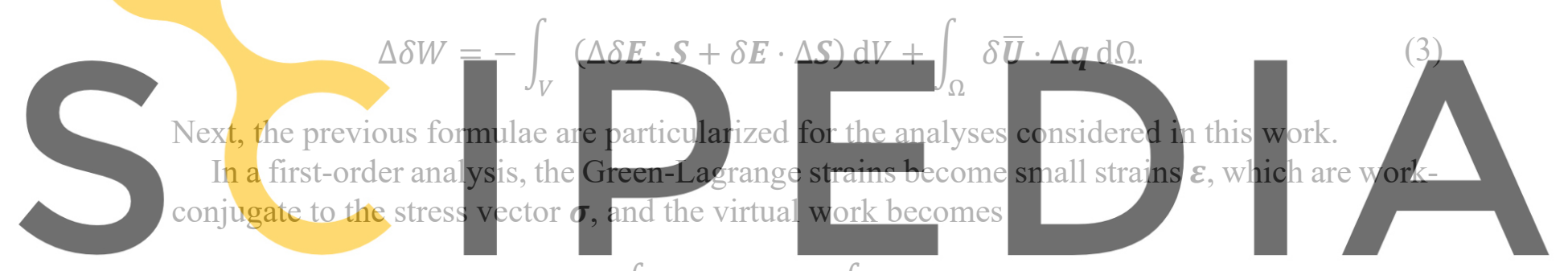
$\delta W=-\int \delta \varepsilon \cdot \sigma \mathrm{d} V+\int \delta \bar{U} \cdot q \mathrm{~d} \Omega$

(4)

Register for free at https//www.scipedia.com to download the version without the watermark

In the elastic case, $\sigma=C \varepsilon$ and the first term gives the element linear stiffness matrix, recalling that no linearization is required, and the second term yieids the element nodai force vector. For elastoplastic materials, Eq. (2) is used and the linearization of Eq. (4) reads

$$
\Delta \delta W=-\int_{V} \delta \boldsymbol{\varepsilon} \cdot \boldsymbol{C}_{c t} \Delta \boldsymbol{\varepsilon} \mathrm{d} V+\int_{\Omega} \delta \overline{\boldsymbol{U}} \cdot \Delta \boldsymbol{q} \mathrm{d} \Omega
$$

In a bifurcation (linear stability) analysis, an initial first-order analysis must be performed to retrieve the pre-buckling stresses. Since this paper focuses on thin-walled members, only membrane stresses $\boldsymbol{S}^{M}$ are kept. The linearization of Eq. (3) at the initial configuration yields

$$
\Delta \delta W(\boldsymbol{U}=\mathbf{0}, \lambda)=-\int_{V}\left(\delta \boldsymbol{\varepsilon} \cdot \boldsymbol{C} \Delta \boldsymbol{\varepsilon}+\Delta \delta \boldsymbol{E} \cdot \lambda \boldsymbol{S}^{M}\right) \mathrm{d} V,
$$

where $\lambda$ is the loading multiplier ( $\boldsymbol{S}^{M}$ corresponds to $\lambda=1$ ). The first and second terms in Eq. (6) lead to the finite element linear and geometric stiffness matrices, respectively. The eigenvalue problem resulting from the singularity of the total stiffness matrix gives the bifurcation loads and associated buckling modes. 
The finite element procedures were implemented in MATLAB [22]. A post-processing module was built to visualize and analyze the deformed/eigenmode shapes obtained.

\subsection{The MITC-4 shell finite element}

Following previous work [23,24], the shell element selected is based on the ReissnerMindlin assumption and is designated as MITC-4 (4-noded Mixed Interpolation of Tensorial Components) $[25,26]$, but it is stressed that any other shell element could have been used. Fig. 1 displays three reference systems: (i) the global Cartesian system $(X, Y, Z)$, (ii) the element Cartesian local axes $(x, y, z)$, where $x$ is aligned with one of the lateral faces and $z$ defines the through-thickness direction (the shell is initially flat), and (iii) a convected frame $(r, s, t)$, where $t$ coincides with the local $z$ axis. The displacement field $\boldsymbol{U}$ is written as a function of the midsurface displacement components $u, v, w$ and the rotations $\theta_{x}, \theta_{y}$, along the local axes, i.e.,

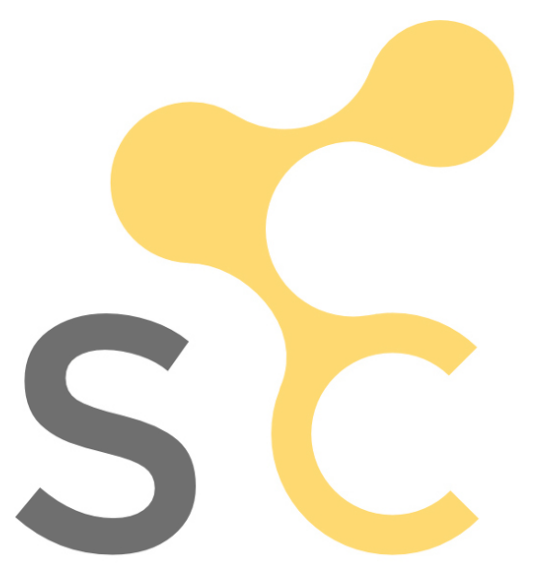

$$
U(x, y, z)=\left[\begin{array}{c}
U_{x} \\
U_{y} \\
U_{z}
\end{array}\right]=\left[\begin{array}{c}
u(x, y)+z \theta_{y}(x, y) \\
v(x, y)-z \theta_{x}(x, y) \\
W(x, y)
\end{array}\right] .
$$

\section{Register for free at https//www.scipedia.com to download the version without the watermark}

The Green-Lagrange strains are subdivided into membrane $(M)$, bending $(B)$ and throughthickness shear $(S)$ components, but only the non-linear part of $\boldsymbol{E}^{M}$ is kept,

$$
\begin{gathered}
\boldsymbol{E}^{M}=\left[\begin{array}{c}
E_{x x}^{M} \\
E_{y y}^{M} \\
2 E_{x y}^{M}
\end{array}\right]=\left[\begin{array}{c}
u_{, x} \\
v_{, y} \\
u_{, y}+v_{, x}
\end{array}\right]+\frac{1}{2}\left[\begin{array}{c}
\overline{\boldsymbol{U}}_{, x} \cdot \overline{\boldsymbol{U}}_{, x} \\
\overline{\boldsymbol{U}}_{, y} \cdot \overline{\boldsymbol{U}}_{, y} \\
2 \overline{\boldsymbol{U}}_{, x} \cdot \overline{\boldsymbol{U}}_{, y}
\end{array}\right], \\
\boldsymbol{E}^{B}=\left[\begin{array}{c}
E_{x x}^{B} \\
E_{y y}^{B} \\
2 E_{x y}^{B}
\end{array}\right]=z\left[\begin{array}{c}
\theta_{y, x} \\
-\theta_{y, x} \\
\theta_{y, y}-\theta_{x, x}
\end{array}\right], \\
\boldsymbol{E}^{S}=\left[\begin{array}{l}
2 E_{x z} \\
2 E_{y z}
\end{array}\right]=\left[\begin{array}{l}
w_{, x}+\theta_{y} \\
w_{, y}-\theta_{x}
\end{array}\right],
\end{gathered}
$$


with $\overline{\boldsymbol{U}}^{T}=\left[\begin{array}{lll}u & v & w\end{array}\right]$. Shear locking is circumvented by re-interpolating the covariant through-thickness shear strains from their values at the so-called "tying points" [25]. The finite element is obtained by interpolating the mid-surface displacements and rotations using linear functions and following a standard isoparametric procedure. The independent kinematic parameters are collected in vector

$$
\boldsymbol{\phi}^{T}=\left[\begin{array}{lllll}
u & v & w & \theta_{x} & \theta_{y}
\end{array}\right],
$$

and are interpolated through $\boldsymbol{\phi}=\boldsymbol{\Psi d}$, where matrix $\boldsymbol{\Psi}$ contains the interpolation functions

$$
\boldsymbol{\Psi}^{T}=\left[\begin{array}{lllll}
\boldsymbol{\psi}_{u}^{T} & \boldsymbol{\psi}_{v}^{T} & \boldsymbol{\psi}_{w}^{T} & \boldsymbol{\psi}_{\theta_{x}}^{T} & \boldsymbol{\psi}_{\theta_{y}}^{T}
\end{array}\right]
$$

where sub-matrices $\boldsymbol{\psi}_{(\cdot)}$ are organised to match the vector of nodal unknowns components,

$$
\boldsymbol{d}^{T}=\left[\begin{array}{lllllllllll}
u_{1} & v_{1} & w_{1} & \left(\theta_{x}\right)_{1} & \left(\theta_{y}\right)_{1} & \cdots & u_{4} & v_{4} & w_{4} & \left(\theta_{x}\right)_{4} & \left(\theta_{y}\right)_{4}
\end{array}\right],
$$

leading to a 20 DOF element.

In a first-order analysis, recalling Eq. (4), the finite element out-of-balance force vector $\boldsymbol{g}_{e}$ is given by

$$
\boldsymbol{g}_{e}=\int_{V}\left[\begin{array}{c}
\boldsymbol{B}_{M}+\boldsymbol{B}_{B} \\
\boldsymbol{B}_{S}
\end{array}\right]^{T} \boldsymbol{\sigma} \mathrm{d} V-\int_{\Omega} \overline{\boldsymbol{\Psi}}_{U}^{T} \boldsymbol{q} \mathrm{d} \Omega,
$$

where $\bar{\Psi}_{U}^{T}=\left[\begin{array}{lllll}\psi_{u}^{T} & \psi_{\nu}^{T} & \psi_{w}^{T} & 0 & 0\end{array}\right]$ interpolates the mid-surface displacements (i.e. $\bar{U}=$ $\left.\bar{\Psi}_{U} d\right)$ and the expressi while $B_{S}$ is presented in vector $\Delta f_{e}$ are obtained
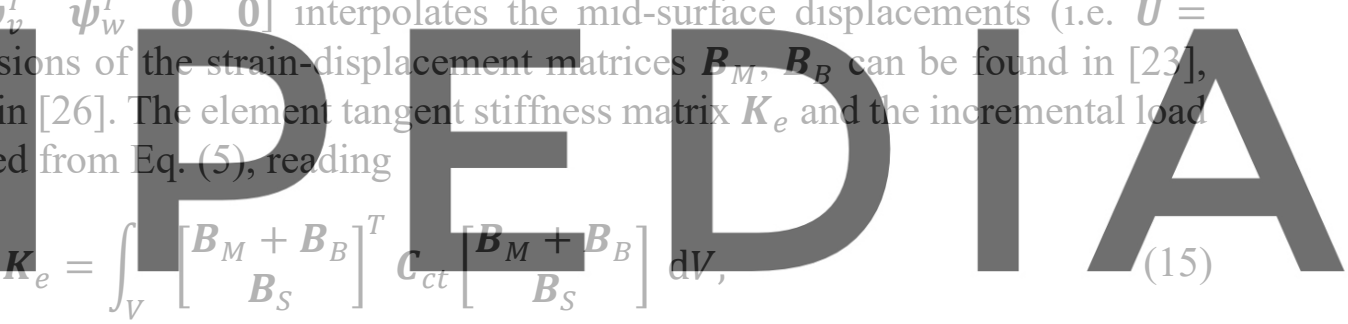

Register for free at https//www.scipedia.com to download the version without the watermark

$$
\Delta f_{e}=\int_{\Omega} \bar{\Psi}_{U}^{T} \Delta q \mathrm{~d} \Omega
$$

For an elastic material, a plane stress state is assumed $\left(\sigma_{z z}=0\right)$, and a St. Venant-Kirchhoff material law is adopted, with

$$
\boldsymbol{C}_{c t}=\boldsymbol{C}=\left[\begin{array}{ccccc}
\frac{E}{1-v^{2}} & \frac{v E}{1-v^{2}} & 0 & 0 & 0 \\
\frac{v E}{1-v^{2}} & \frac{E}{1-v^{2}} & 0 & 0 & 0 \\
0 & 0 & G & 0 & 0 \\
0 & 0 & 0 & G & 0 \\
0 & 0 & 0 & 0 & G
\end{array}\right] \text {, }
$$

where $E$ and $G$ are Young's and shear moduli and $v$ is Poisson's ratio. For an elastoplastic material, small-strain $J_{2}$ plasticity is adopted, with an associated flow rule and no hardening. The yield function is thus given by 


$$
f=\sqrt{3 / 2}\|\operatorname{dev}(\boldsymbol{\sigma})\|-\sigma_{0},
$$

where $\|\operatorname{dev}(\boldsymbol{\sigma})\|$ is the norm of the deviatoric stresses and $\sigma_{0}$ is the uniaxial yield stress. At the end of each iteration, elastoplastic stresses are updated using the backward-Euler return with the $\sigma_{z z}=0$ constraint. The quadratic convergence of the iterative procedure is ensured by using the consistent tangent constitutive operator $\boldsymbol{C}_{c t}$ (see, e.g., [21,27]).

In a bifurcation (linear stability) analysis, the element geometric matrix is obtained from the second term of Eq. (6), leading to

$$
\boldsymbol{G}_{e}=\lambda \int_{V} S_{x x}^{M} \overline{\boldsymbol{B}}_{x x}^{T} \overline{\boldsymbol{B}}_{x x}+S_{y y}^{M} \overline{\boldsymbol{B}}_{y y}^{T} \overline{\boldsymbol{B}}_{y y}+S_{x y}^{M}\left(\overline{\boldsymbol{B}}_{x x}^{T} \overline{\boldsymbol{B}}_{y y}+\overline{\boldsymbol{B}}_{y y}^{T} \overline{\boldsymbol{B}}_{x x}\right) \mathrm{d} V,
$$

where the auxiliary matrices $\overline{\boldsymbol{B}}_{x x}$ and $\overline{\boldsymbol{B}}_{y y}$ are provided in [23].

For the computation of all matrices and vectors, numerical integration is carried out with a $2 \times 2$ Gauss point grid in the shell mid-surface. Along the thickness direction, for the elastic case, analytical integration is performed, whereas in the elastoplastic case 6 Gauss points are used, to ensure that the plasticity spreading is captured accurately.

\subsection{The GBT-based finite element}

The GBT element implemented corresponds to that proposed in [20], although (i) geometrical imperfections are not considered, (ii) some simplifications are introduced to the membrane stress and strain fields, (iii) a linear elastic material is always gssumed and (iv) the
only non-linear strain terms kept in a bifurcation analysis are the longitudinal membrane one
(acceptable for slender bars).
Fig. 2 shows two walls of an arbitrary prisinatic thin-walled bar, the global Cartesian axes
$(X, Y, Z)$, where $X$ defines the member longitudinal axis, and the local Cartesian axes for each wall $(x, y, z)$, defining the member axis, wall mid-line and thickness directions, respectively.

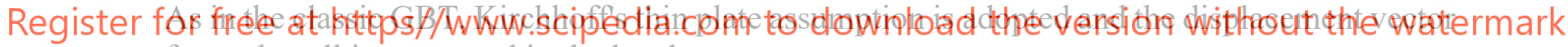
for each wall is expressed in the local axes as

$$
\boldsymbol{U}(x, y, z)=\left[\begin{array}{l}
U_{x} \\
U_{y} \\
U_{z}
\end{array}\right]=\left[\begin{array}{c}
(\overline{\boldsymbol{u}}-z \overline{\boldsymbol{w}}) \cdot \boldsymbol{\phi}, x \\
(\overline{\boldsymbol{v}}-z \overline{\boldsymbol{w}}, y) \cdot \boldsymbol{\phi} \\
\overline{\boldsymbol{w}} \cdot \boldsymbol{\phi}
\end{array}\right],
$$

where $\overline{\boldsymbol{u}}(y), \overline{\boldsymbol{v}}(y), \overline{\boldsymbol{w}}(y)$ are column vectors containing the displacement components of the GBT "cross-section deformation modes" along $x, y$ and $z$, respectively, and $\phi(x)$ is a column vector collecting their amplitude functions (the problem unknowns). These deformation modes are obtained from a "GBT cross-section analysis" [28,29], implemented in the GBTUL program [30], available at www.civil.ist.utl.pt/gbt. This analysis is based on a cross-section discretization into "natural" nodes, located at wall mid-line intersections and free ends, and "intermediate nodes", arbitrarily located within each wall mid-line. 


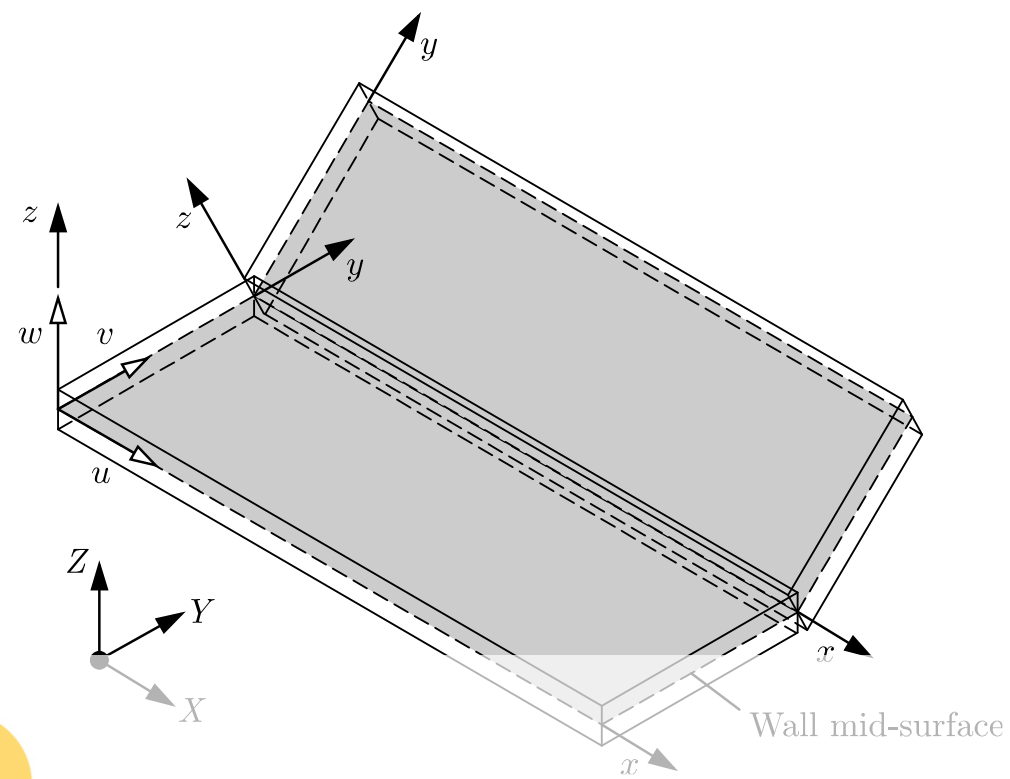

Figure 2: Arbitrary thin-walled bar: global/local coordinate systems and local displacement components.

In the first-order case, the non-null small strain components read

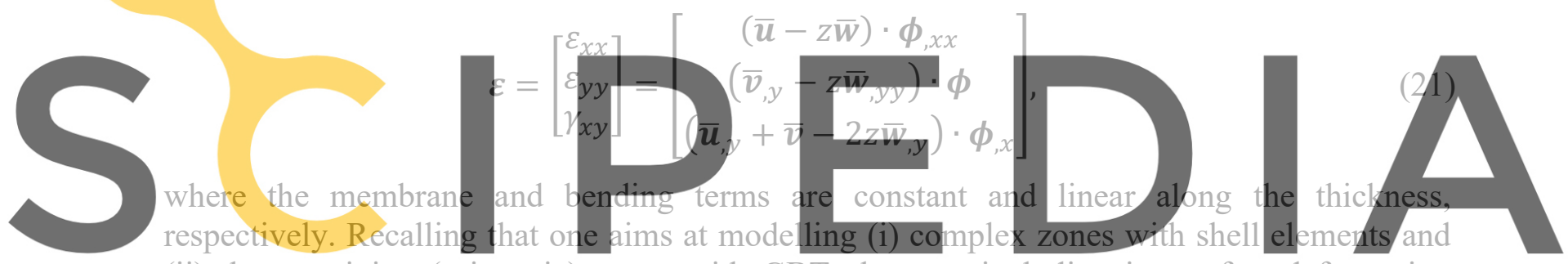

(ii) the remaining (prismatic) zones with GBT elements including just a few deformation

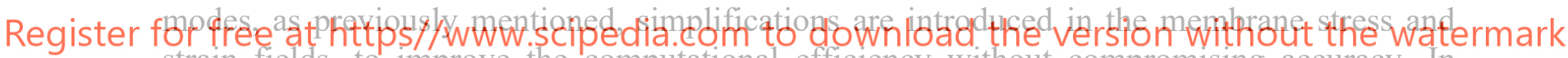
strain fields, to improve the computational efficiency without compromising accuracy. In particular, it is assumed that $\varepsilon_{y y}^{M}=\sigma_{x y}^{M}=0$ and $\gamma_{x y}^{M}=\sigma_{x y}^{M}=0$. With these constraints, only two deformation mode sets are obtained: (i) the so-called natural Vlasov modes (axial extension, bending, torsion for open sections and distortional modes) and (ii) local-plate modes. In closed sections, the torsional mode involves membrane shear strains and, therefore, the $\gamma_{x y}^{M}=$ 0 constraint must be relaxed (for this mode only).

As stated before, in a bifurcation analysis only the non-linear longitudinal membrane strains are kept, meaning that the Green-Lagrange strains read $\boldsymbol{E}^{T}=\left[\begin{array}{lll}E_{x x} & \varepsilon_{y y} & \gamma_{x y}\end{array}\right]^{T}$, with

$$
E_{x x}^{M}=\varepsilon_{x x}+\frac{1}{2}\left(\boldsymbol{\phi}_{, x}^{T}\left(\overline{\boldsymbol{v}} \overline{\boldsymbol{v}}^{T}+\overline{\boldsymbol{w}} \overline{\boldsymbol{w}}^{T}\right) \boldsymbol{\phi}_{, x}+\boldsymbol{\phi}_{, x x}^{T} \overline{\boldsymbol{u}} \overline{\boldsymbol{u}}^{T} \boldsymbol{\phi}_{, x x}\right) .
$$

Furthermore, the term $\overline{\boldsymbol{u}} \overline{\boldsymbol{u}}^{T}$ is also discarded without significant loss of accuracy [15].

A plane stress state is assumed, with $\boldsymbol{S}^{T}=\left[S_{x x} S_{y y} S_{x y}\right]^{T}$. Due to the $\varepsilon_{y y}^{M}=0$ constraint, the membrane and bending stress components are separated to avoid over-stiff solutions, with

$$
\boldsymbol{S}^{M}=\boldsymbol{C}^{M} \boldsymbol{E}^{M}, \quad \boldsymbol{S}^{B}=\boldsymbol{C}^{B} \boldsymbol{E}^{B},
$$




$$
\boldsymbol{C}^{M}=\left[\begin{array}{lll}
E & 0 & 0 \\
0 & 0 & 0 \\
0 & 0 & 0
\end{array}\right], \quad \boldsymbol{C}^{B}=\frac{E}{1-v^{2}}\left[\begin{array}{ccc}
1 & v & 0 \\
v & 1 & 0 \\
0 & 0 & \frac{1-v}{2}
\end{array}\right]
$$

The finite element is obtained by interpolating the amplitude functions using Hermite cubic polynomials for all deformation modes except those involving only warping (e.g., axial extension), in which case quadratic hierarchical Lagrange functions are adopted. In accordance with the shell element interpolation, $\boldsymbol{\phi}=\boldsymbol{\Psi} \boldsymbol{d}$, with the matrix of the interpolation functions $\Psi$ and the vector of nodal unknowns $\boldsymbol{d}$.

In a linear analysis, from Eq. (4), the element stiffness matrix reads

$$
\boldsymbol{K}_{e}=\int_{L}\left[\begin{array}{c}
\boldsymbol{\Psi} \\
\boldsymbol{\Psi}_{, x} \\
\Psi_{, x x}
\end{array}\right]^{T}\left[\begin{array}{ccc}
\mathbf{B} & \mathbf{0} & \mathbf{D}_{2} \\
\mathbf{0} & \mathbf{D}_{1} & \mathbf{0} \\
\mathbf{D}_{2}^{T} & 0 & \mathbf{C}
\end{array}\right]\left[\begin{array}{c}
\boldsymbol{\Psi} \\
\boldsymbol{\Psi}_{, x} \\
\Psi_{, x x}
\end{array}\right] \mathrm{d} x
$$

where $L$ is the finite element length and the GBT modal matrices are given by ( $S$ is the crosssection mid-line)

$$
\mathbf{B}=\int_{S} \frac{E h^{3}}{12\left(1-v^{2}\right)} \overline{\boldsymbol{w}}_{, y y} \overline{\boldsymbol{w}}_{, y y}^{T} \mathrm{~d} y, \quad \mathbf{C}=\int_{S} E h \overline{\boldsymbol{u}}^{T} \overline{\boldsymbol{u}}^{T}+\frac{E h^{3}}{12\left(1-v^{2}\right)} \overline{\boldsymbol{w}}^{T} \overline{\boldsymbol{w}}^{T} \mathrm{~d} y,
$$
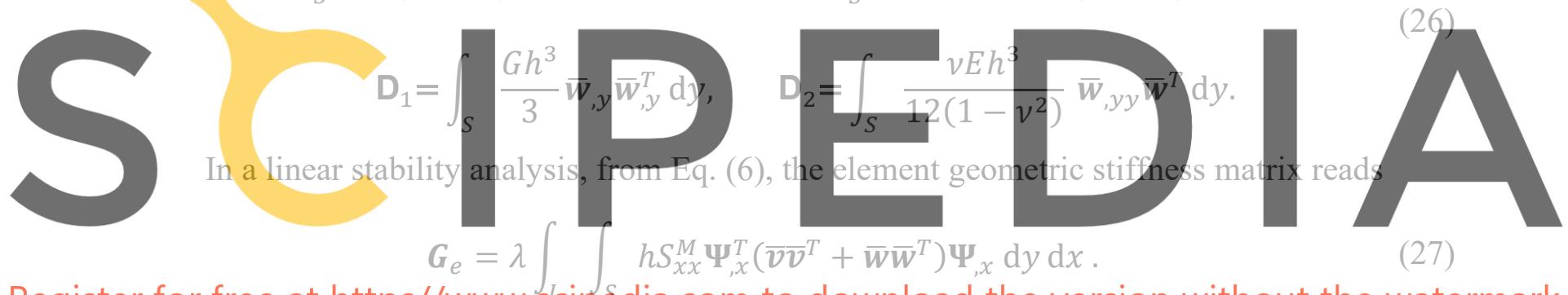

\section{Register for free at https//www.scipedia.com to download the version without the watermark}

Finally, in the elastoplastic case, recall that the GBT element stiffness matrix is assumed elastic. The incremental load vector stems from Eq. (5) and reads

$$
\Delta \boldsymbol{f}_{e}=\int_{\Omega}\left[\begin{array}{c}
\boldsymbol{\Psi} \\
\boldsymbol{\Psi}_{, x}
\end{array}\right]^{T}\left[\begin{array}{ccc}
\mathbf{0} & \overline{\boldsymbol{v}} & \overline{\boldsymbol{w}} \\
\overline{\boldsymbol{u}} & \mathbf{0} & \mathbf{0}
\end{array}\right] \Delta q \mathrm{~d} \Omega
$$

All these matrices and vectors are integrated analytically, with the exception of (i) $\boldsymbol{G}_{e}$, in which case a $3 \times 3$ mid-surface Gauss point grid is adopted in each wall and analytical integration is performed along the thickness, and (ii) the elastoplastic case, with $3 \times 3$ midsurface and 2 through-thickness Gauss points.

\section{PROCEDURE TO COMBINE SHELL AND GBT-BASED FINITE ELEMENTS}

As already pointed out, in the proposed approach shell finite elements are used in zones with complex geometries and/or non-linear material behaviour, whereas GBT elements are adopted for the prismatic and elastic zones, where just a few deformation modes lead to accurate results. At a shell-GBT interface, compatibility is enforced through constraint equations of the form 


$$
\zeta_{j}\left(U_{k j}^{S F E}(z=0)-U_{k j}^{G B T}(z=0)\right)=0,
$$

where $\zeta_{j}$ are Lagrange multipliers and $U_{k j}^{S F E}, U_{k j}^{G B T}$ are the displacements of the mid-surface $(z=0)$ node $k$ along direction $j$, in the shell and GBT finite elements, respectively. Since only mid-surface nodal displacements are constrained, compatibility is not fully guaranteed across the interface, as the shell elements adopt a Reissner-Mindlin description, whereas GBT is based on Kirchhoff's assumption. However, this issue is not significant for thin-walled members, since plate-like shearing has little importance. If this effect plays a significant role, the shell zones must be extended (the illustrative examples will discuss this issue). Normally, the number of shell DOFs is equal or higher than the GBT one at the interface and the constraint equations can be written as

$$
\left[\begin{array}{l}
\boldsymbol{\zeta}_{c} \\
\zeta_{d}
\end{array}\right]^{T}\left(\left[\begin{array}{lll}
\mathbf{0} & \mathbf{1} & \mathbf{0} \\
0 & 0 & 1
\end{array}\right]\left[\begin{array}{l}
\boldsymbol{d}_{u}^{S F E} \\
d_{c}^{S F E} \\
d_{d}^{S F E}
\end{array}\right]-\left[\begin{array}{cc}
\mathbf{0} & \boldsymbol{B}_{C C}^{G B T} \\
0 & B_{d c}^{G B T}
\end{array}\right]\left[\begin{array}{l}
\boldsymbol{d}_{u}^{G B T} \\
d_{c}^{G B T}
\end{array}\right]\right)=0,
$$

where subscripts $u, c$ and $d$ denote DOFs that are, respectively, "unconstrained" (do not appear in the equations), "constrained" (associated to the "linked nodes" in Fig. 3, which means that $\boldsymbol{B}_{c C}^{G B T}$ is a square and invertible matrix) and "dependent" (corresponding to the "hanging nodes" in Fig. 3). Matrices $\boldsymbol{B}_{i j}^{G B T}$ are calculated by obtaining the deformation modes with GBTUL and

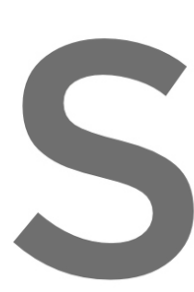
then using Eq. (20) to determi

If the axes are not coinc

Within an increment

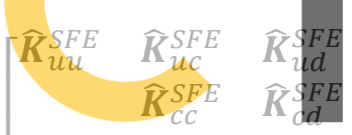

$\widehat{\boldsymbol{K}}_{d d}^{S F E}$
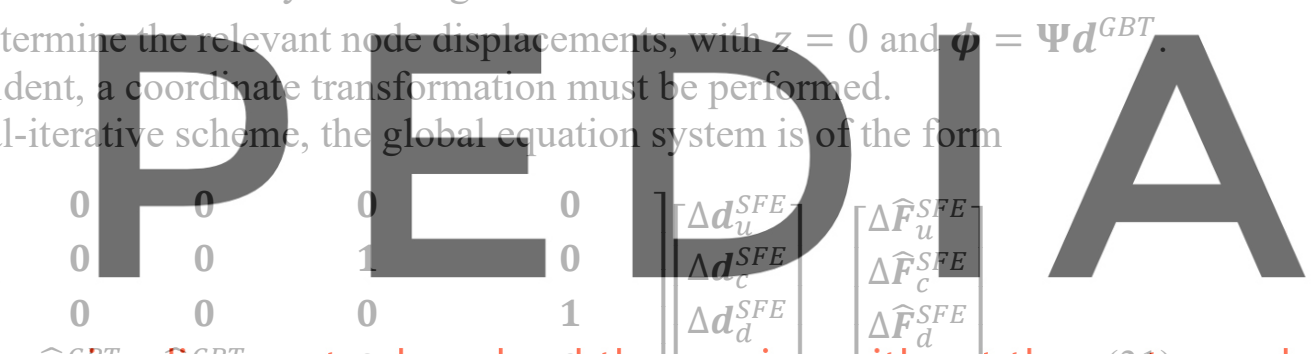

Register for free at https//www

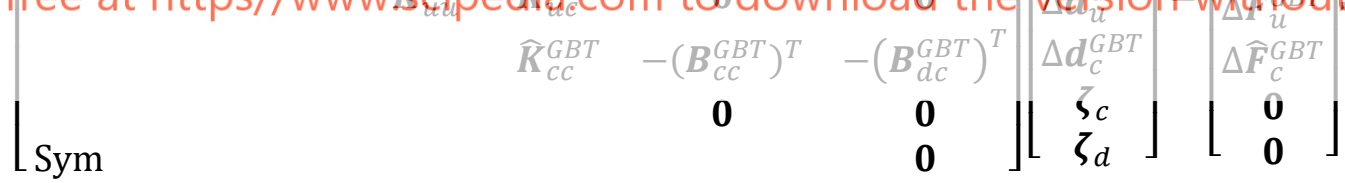

where the expressions for $\widehat{\boldsymbol{K}}_{i j}, \Delta \boldsymbol{d}_{i}$ and $\Delta \widehat{\boldsymbol{F}}_{i}$ depend on the analysis type and are discussed next.

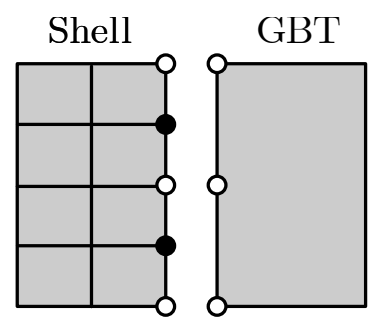

- hanging node

o linked node

Figure 3: Interface between shell elements and a GBT element (only a single wall is shown). 


\subsection{First-order analysis}

In a first-order analysis, the variations $\Delta$ and the "hats" $\left({ }^{-}\right)$are dropped and Eq. (31) can be simplified by eliminating the Lagrange multipliers and condensing the shell DOFs, leading to a system that involves only the GBT DOFs. Besides leading to a significant DOF economy, this procedure is advantageous if a shell macro-element library is available (i.e., matrices $\boldsymbol{K}_{i j}^{S F E}$ are available for several types of connections) and/or the macro-elements are repeated at several locations in the model (typical in steel structures). In this case the hanging node DOFs $\boldsymbol{d}_{d}^{S F E}$ are not constrained, since it is assumed that no severe localized effects occur at the GBT-shell interface. If this assumption is not reasonable, either these displacements are constrained or the shell zone is extended.

\subsection{Physically non-linear analysis}

In a physically non-linear (but geometrically linear) analysis, the "hats" ( $\widehat{(})$ are again dropped, the tangent stiffness matrices for the shell elements, $\mathbb{K}_{i j}^{S F E}$, are calculated using $C_{c t}$ and $\Delta \boldsymbol{F}_{\boldsymbol{i}}$ are replaced by the out-of-balance force vectors $\boldsymbol{g}_{i}\left(\boldsymbol{g}_{i}^{G B T}=\mathbf{0}\right.$ except for the first iteration of each increment). The Lagrange multipliers can be eliminated from Eq. (31) and, since the GBT elements are elastic, they are evaluated only once and the corresponding DOFs can be condensed. The shell DOFs could be condensed instead, but this requires performing the related operations in all iterations. In such case, $d_{d}^{S F E}$ needs to be constrained, otherwise stress concentrations appear near the linked nodes and disturb the stress flow through the GBT-shdl interface.

To optimize the computational cost, an as first step, GBT (elastic) elements are used in the convergence is achieved, a subroutine checks whether the integration point stress es of the GBT elements fall outside the yield surface. If this occurs, the corresponding GBT element is re-

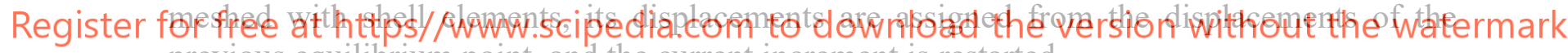
previous equilibrium point, and the current increment is restarted.

\subsection{Linear stability analysis}

In a linear stability analysis, the corresponding eigenvalue problem is straightforwardly obtained from Eq. (31), with $\Delta \widehat{\boldsymbol{F}}_{i}=\mathbf{0}, \widehat{\boldsymbol{K}}_{i j}=\boldsymbol{K}_{i j}+\boldsymbol{G}_{i j}$, where $\boldsymbol{G}_{i j}$ are geometric stiffness matrices.

\section{NUMERICAL EXAMPLES}

In this section a set of selected examples are presented and discussed, to demonstrate the capabilities and potential of the proposed approach. In all examples, the results obtained with the proposed approach and full MITC-4 shell finite element models are compared.

\subsection{Lipped channel cantilever with a tapered segment}

The first example concerns a lipped channel cantilever with a linearly tapered segment. Fig. 4(a) shows the beam geometry, material parameters and GBT cross-section discretization 
adopted. With this discretization, 39 deformation modes are obtained with GBTUL but, as already mentioned in Section 2.2, only the natural Vlasov (1 to 6) and local-plate modes (7 to 15) are included in the analyses (see Fig. 4(b)).

Three models are considered: (i) a full shell model with 2220 elements, (ii) a less refined GBT-shell model, where only the tapered zone is discretized with shell elements (550 shell elements +10 GBT elements), and (iii) a refined GBT-shell model where the shell zone is extended $0.25 \mathrm{~m}$ into the uniform zone (1100 shell elements +5 GBT elements).
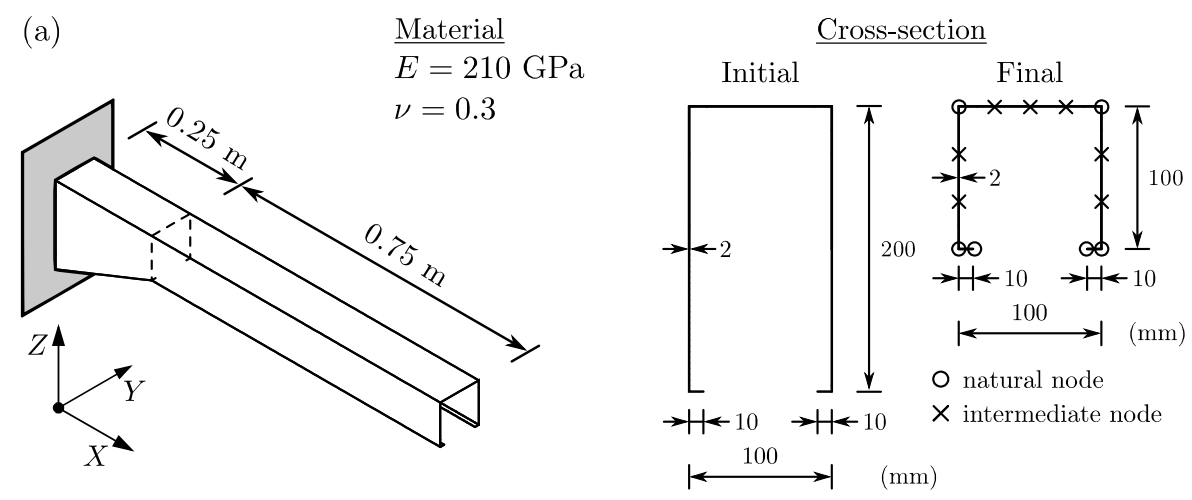

(b)

Vlasov modes

Local-plate modes (In-plane only)
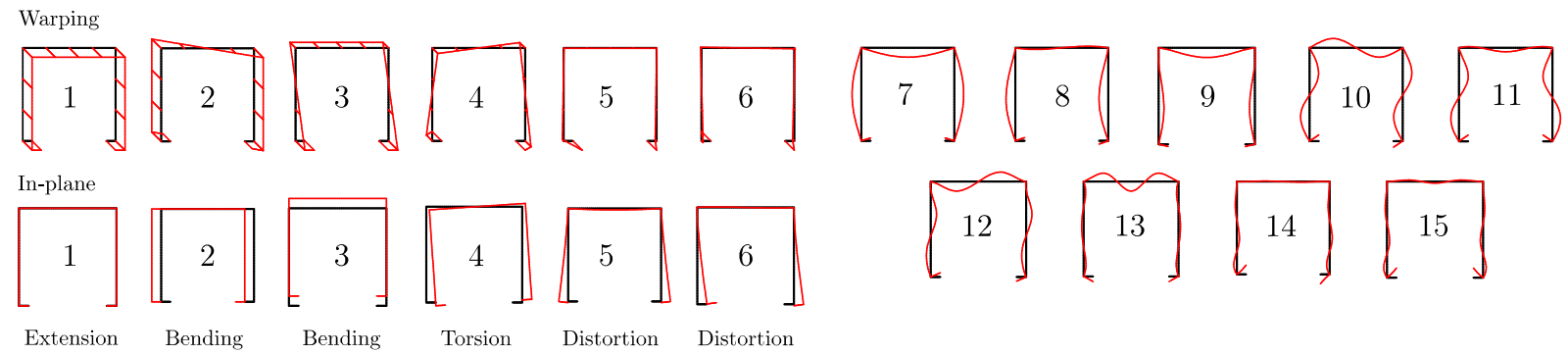

Figure 4: Lipped channel cantilever: (a) geometry, material parameters and GBT cross-section discretization, and (b) first 15 cross-section deformation modes.

Fig. 5(a) displays the linear results obtained when two concentrated loads are applied at the free end. Both GBT-shell solutions agree very well with the full shell model, as confirmed by the displacement values of points $\mathrm{P} 1$ and $\mathrm{P} 2$ in the figure (differences below 1\%) and the deformed configurations. The mode amplitude graph shows that minor axis bending (B3) and symmetric distortion (D5) are the most relevant modes. Near the interface zone $(X=0)$ several local-plate (LP) modes appear in the less refined GBT-shell model, although their presence is hardly noticeable in the corresponding graph. The mode amplitude graph of the most refined GBT-shell model is basically a truncated version of the less refined one, resulting in a vanishing of the LP modes. This shows that, as highlighted in Section 3, extending the shell zone dissipates local effects stemming from singularities and, furthermore, the number of deformation modes in the GBT-based element can be reduced - for instance, the LP modes could have been discarded in this model.

Fig. 5(b) shows the first buckling load and associated buckling mode for each model (for a load profile consisting of $2 \times 1 \mathrm{kN}$ loads). The mode amplitude graphs show that the buckling 
(a)

\begin{tabular}{llr}
\hline Model & Point & \multicolumn{1}{c}{ Displacement $(\mathrm{mm})$} \\
\hline MITC4 (2 220 FE) & P1 $\left(U_{Y}\right)$ & \multicolumn{1}{c}{3.0529} \\
& P2 $\left(U_{Z}\right)$ & \multicolumn{1}{c}{-26.7459} \\
MITC4 (1 100 FE) & P1 $\left(U_{Y}\right)$ & $3.0724(0.64 \%)$ \\
+ GBT (5 FE) & P2 $\left(U_{Z}\right)$ & $-26.7208(0.09 \%)$ \\
MITC4 (550 FE) & P1 $\left(U_{Y}\right)$ & $3.0258(0.89 \%)$ \\
+ GBT (10 FE) & P2 $\left(U_{Z}\right)$ & $-26.9295(0.69 \%)$ \\
\hline
\end{tabular}

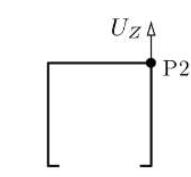

$X=1.0 \mathrm{~m}$
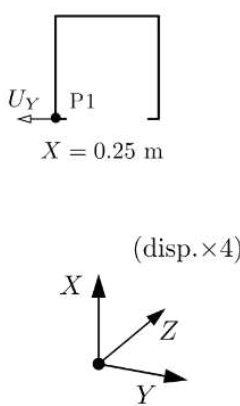

(b)
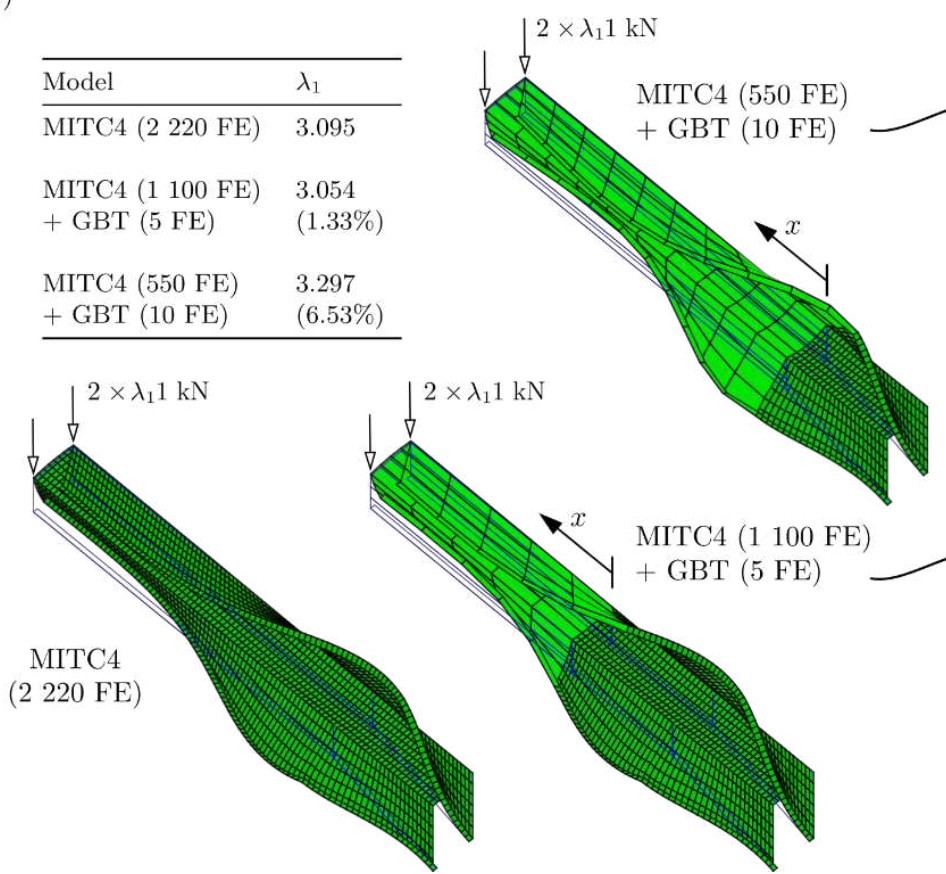

MITC4 (1 $100 \mathrm{FE})$

$+\mathrm{GBT}(5 \mathrm{FE})$

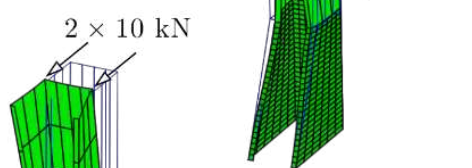

Mode amplitude (modes in $\mathrm{cm}$ )

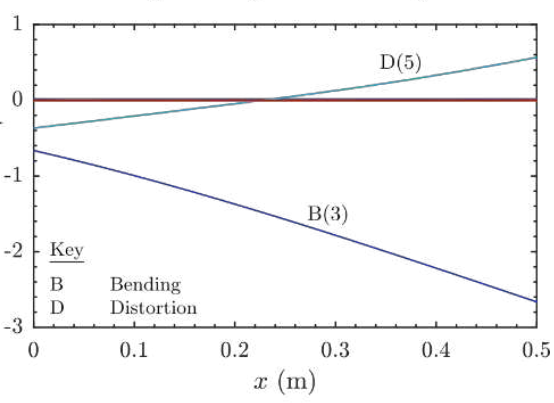

Mode amplitude

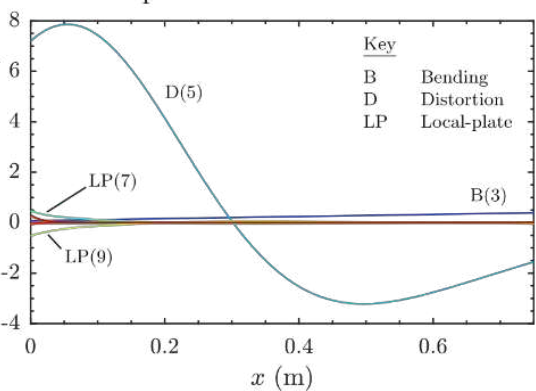

Mode amplitude

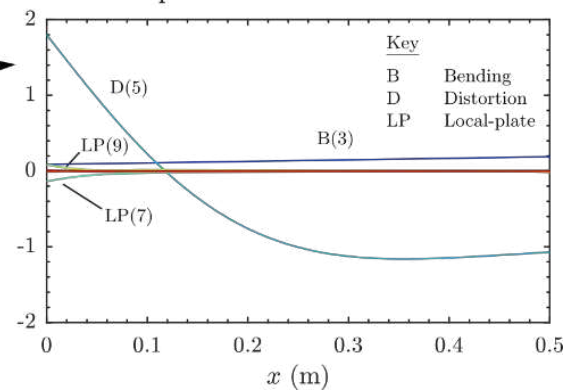

Figure 5: Lipped channel cantilever: (a) linear analysis and (b) bifurcation analysis (first buckling load). 
modes are essentially distortional (D5), although minor-axis bending (B3) also participates along the beam and LP modes appear near the GBT-shell transition zone. The refined GBTshell model leads to a bifurcation load nearly coincident with that of the full shell model (the difference is less than $2 \%$ ), illustrating the improvement that can be achieved by extending the shell macro-element beyond the transition zone (the relevance of the LP modes is reduced). Note that the buckling load obtained with the refined GBT-shell model is slightly lower than the full shell model one, due to the lack of compatibility along the GBT-shell interface.

\subsection{Lipped channel cantilever with two long holes}

The second example consists of a lipped channel cantilever with the same material parameters and cross-section of the prismatic segment of the previous example (see Fig. 4(a)) but having two long holes located in the middle of each flange. Fig. 6(a) displays the beam geometry and loadings: (i) a $1 \mathrm{kN}$ lateral ( $-Y$ direction) load applied at one of the flange-lip corners near mid span, for the linear analysis, and (ii) a $1 \mathrm{kN}$ load ( $-Z$ direction) located at the flange-web corner of the free end section, for the bifurcation analysis.

Three models are considered: (i) a shell model without the holes, to assess their influence (1650 elements), (ii) a shell model with the holes (1590 elements) and (iii) a GBT-shell model with shell elements in the $0.55 \mathrm{~m}$ central segment of the beam (660 shell +10 GBT elements).

Fig. 6(b) reports the linear analysis results. A comparison between the shell models with and without holes shows that the latter exhibits a much stiffer response, providing a $73 \%$ lower lateral displacement of the point of load application. The GBT-shell model captures the shell model (with holes) deformed configuration very accurately, with a small difference $(2 \%)$ in terms of the displacement of the point of load application. The modal participation diagrams provided show that the zones without the holes undergo essentially major-axis bending (B2), torsion and a combination of symmetric (D5) and anti-symmetric (D6) distortion. The contributions of local-plate modes are negligible.

Fig. 6(c) displays the bifurcation analysis results (critical loads and associated buckling mode shapes). The holes play once more a very significant role, namely in the critical load value, which is more than $20 \%$ higher in their absence, and in the corresponding buckling mode shapes: (i) with holes, the mode is characterised by a pronounced distortion in the right hole zone, with a significant lateral displacement of the lip, whereas (ii) without holes, cross-section distortion mostly occurs near the support. The GBT-shell model captures the buckling mode quite accurately and the critical load differs by only $2 \%$ with respect to the shell model value.

The modal participation diagrams concerning the buckling mode make it possible to observe that both segments without holes essentially undergo distortion (D5 and D6), even though bending and torsion also appear at the free end zone.

\subsection{Elastoplastic I-section plane frame}

The last example consists of the physically non-linear analysis of the symmetric plane frame shown in Fig. 7(a), which is acted by a point load applied at the top of the mid-span crosssection. At the load application point, $5 \mathrm{~mm}$ thick stiffening plates are provided. Taking advantage of the problem symmetry, only half of the frame is analysed. This example aims at showing that zones with localised plastic strains are easily handled with the proposed approach. 


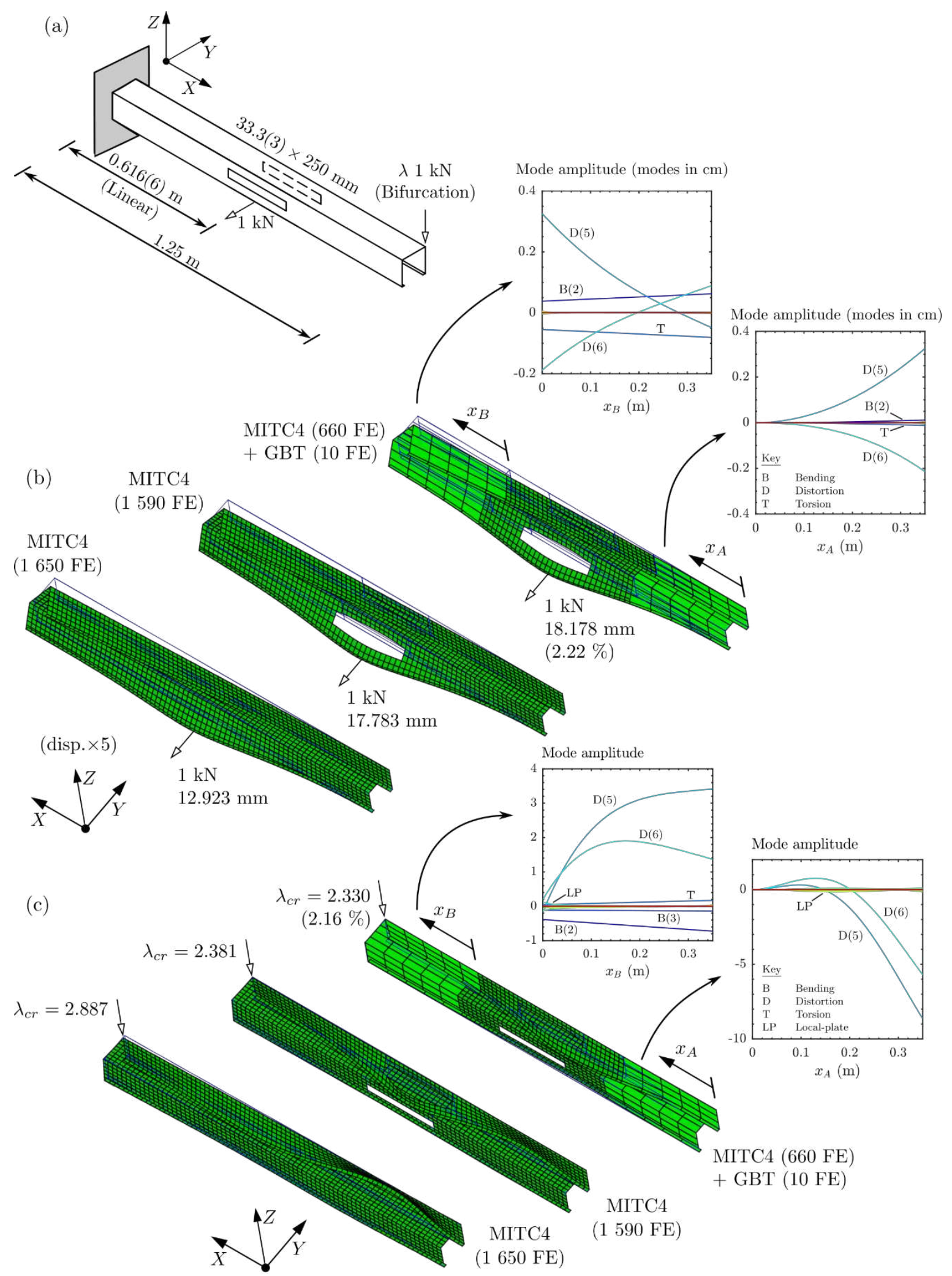

Figure 6: Lipped channel cantilever with two long holes: (a) material parameters, geometry and loading, (b) first-order analysis and (c) bifurcation analysis (first buckling load). 
(a)

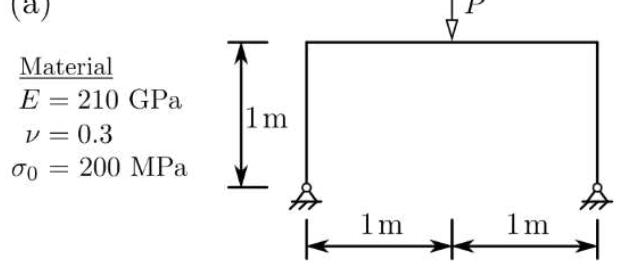

(b)

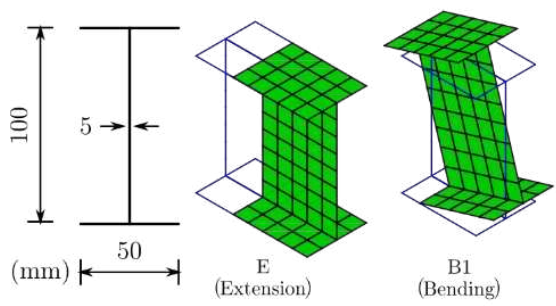

(c)

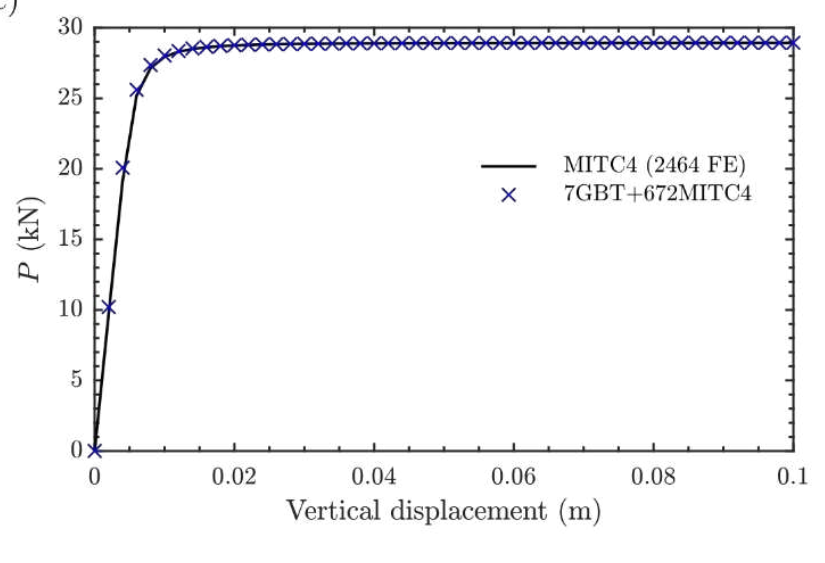

(d)

Mode amplitude (modes in $\mathrm{cm}$ )
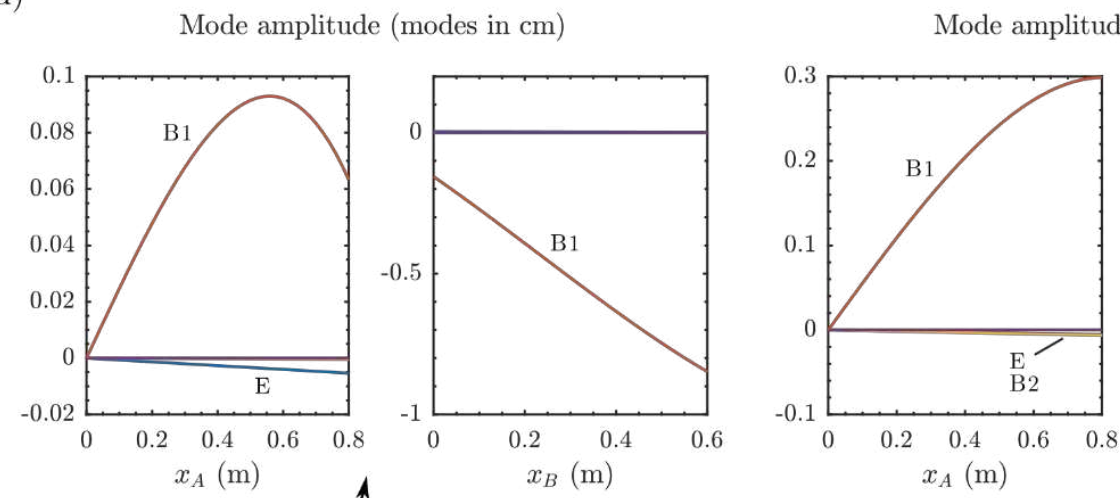

Mode amplitude (modes in $\mathrm{cm}$ )
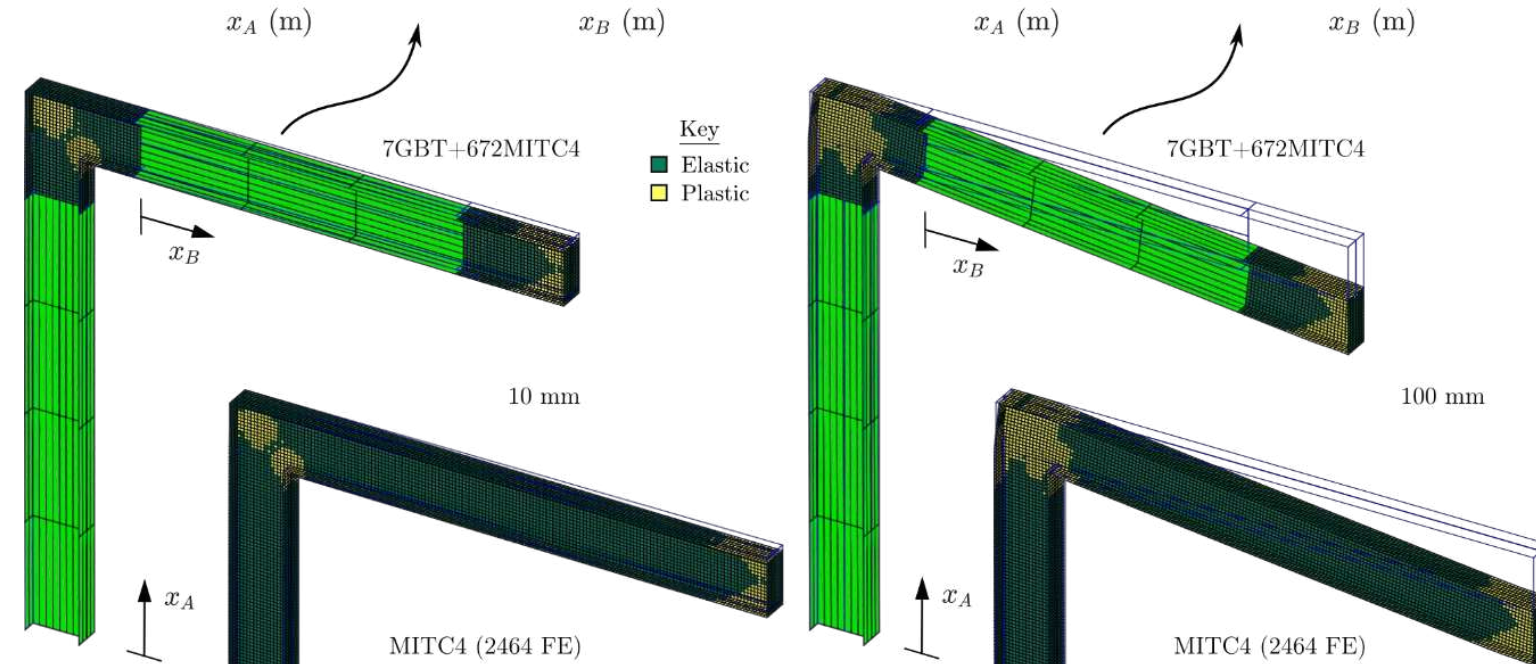

MITC4 (2464 FE)

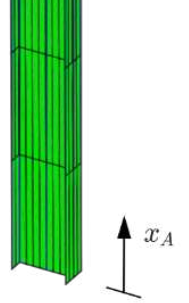

$100 \mathrm{~mm}$
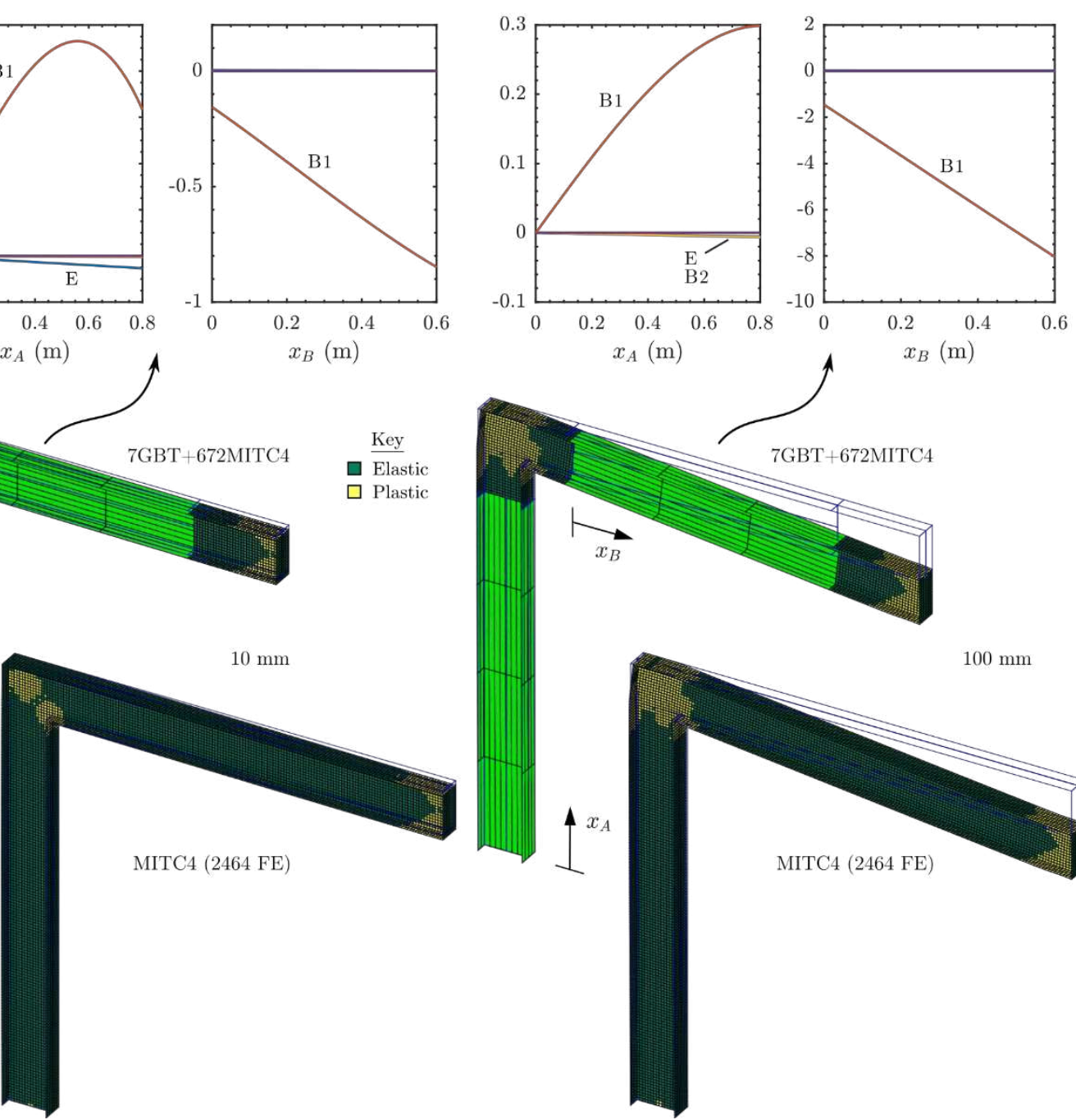

Figure 7: Plane frame: (a) material parameters, geometry and loading, (b) GBT deformation modes,

(c) load-displacement diagram, (d) deformed configurations and mode amplitude graphs. 
Since this is a 2D problem and the cross-section does not have distortional modes, only extension and bending are included in the GBT finite elements (see Fig. 7(b)).

The results obtained with a GBT-shell and a full shell model are shown in Figs. 7(c)-(d): the former plots the load-displacement equilibrium paths and the latter depicts the deformed configurations and the plastic zones for vertical displacements of the point of load application equal to $10 \mathrm{~mm}$ and $100 \mathrm{~mm}$, as well as the corresponding GBT mode amplitude graphs. Once more, an excellent agreement between both models is observed throughout the whole loaddisplacement range considered, even if the number of DOFs is significantly lower in the GBTshell model (recall that the GBT-based finite elements include only two deformation modes). The deformed configurations displayed in the figure make it possible to observe that, throughout the whole displacement range considered, the plastic zones are concentrated at the beam mid-span and beam-to-column connections. The mode amplitude graphs further confirm that major-axis bending is the dominant mode, but small compressive axial forces (corresponding to negative-slope linear amplitudes of the axial extension mode E) develop in the column and beam.

As a final note, it is highlighted that the computation times of the GBT-shell model are about 4 times faster in all steps.

\section{CONCLUSIONS}

The present paper proposed a general and efficient approach to analyse thin-walled members with complex geometries and connections, which combines standard shell and GBT-based finite elements. The GBT elements are used to model the prismatic and elastic members, whereas the more complex zones (with plastic strains, discontinuities, holes, joints, etc.) are handled by means of shell elements. This approach offers noteworthy advantages: (i) the number of deformation modes employed in the GBT elements is greatly reduced (compared to full GBT analyses), (ii) zones with complex geometries (joints, tapered segments, holes, etc.) can be dealt with, (iii) the computational efficiency is maximized, since each element type is employed where it is most effective. Moreover, in many types of analysis, either the shell or GBT DOFs can be condensed out of the global equilibrium equation system, to reduce the number of DOFs and ensure fast computation times - this is particularly important if the full structure has many repetitive zones.

To illustrate the capabilities and potential of the proposed approach, three numerical examples were presented and discussed, concerning linear (first-order), bifurcation (linear stability) and first-order plastic zone analyses. These examples involved tapered segments, holes and frames. For validation and comparison purposes, full shell finite element model solutions were provided and an excellent match was always observed.

Finally, it is worth mentioning that the authors are currently working on extending the approach proposed in this work to analyse thin-walled members and structural systems, so that it covers also the large displacement and finite rotation range.

\section{ACKNOWLEDGMENTS}

The first author gratefully acknowledges the financial support of FCT (Fundação para a Ciência e a Tecnologia, Portugal), through the doctoral scholarship SFRH/BD/130515/2017. 


\section{REFERENCES}

[1] Schardt R. Eine Erweiterung der Technischen Biegetheorie zur Berechnung prismatischer Faltwerke, Stahlbau. (1996) 35(6):161-171. (German)

[2] Schardt R. Verallgemeinerte Technische Biegetheorie, Springer Verlag, Berlin, (1989). (German)

[3] Nedelcu M. GBT formulation to analyse the behaviour of thin-walled members with variable cross-section, Thin-Walled Structures. (2010) 48(8):629-638.

[4] Nedelcu M. GBT formulation to analyse the buckling behaviour of isotropic conical shells, Thin-Walled Structures. (2011) 49(7):812-818.

[5] Muresan A.-A., Nedelcu M., Gonçalves R. GBT-based FE formulation to analyse the buckling behaviour of isotropic conical shells with circular cross-section, Thin-Walled Structures. (2019) 134:84-101.

[6] Cai J., Moen C.D. Elastic buckling analysis of thin-walled structural members with rectangular holes using Generalized Beam Theory, Thin-Walled Structures. (2016) 107:274-286.

[7] Gonçalves R., Camotim D. Improving the efficiency of GBT displacement-based finite elements, Thin-Walled Structures. (2017) 111:165-175.

[8] Peres N., Gonçalves R., Camotim D. First-order Generalised Beam Theory for curved thin-walled members with circular axis, Thin-Walled Structures. (2016) 107:345-361.

[9] Peres N., Gonçalves R., Camotim D. GBT-based cross-section deformation modes for curved thin-walled members with circular axis, Thin-Walled Structures. (2018) 127:769780.

[10] Basaglia C., Camotim D., Silvestre N. Global buckling analysis of plane and space thinwalled frames in the context of GBT, Thin-Walled Structures. (2008) 46(1):79-101.

[11] Basaglia C., Camotim D., Silvestre N. GBT-based local, distortional and global buckling analysis of thin-walled steel frames, Thin-Walled Structures. (2009) 47(11):1246-1264.

[12] Basaglia C., Camotim D., Silvestre N. Post-buckling analysis of thin-walled steel frames using Generalized Beam Theory (GBT), Thin-Walled Structures. (2013) 62:229-242.

[13] Camotim D., Basaglia C. Buckling analysis of thin-walled steel structures using Generalized Beam Theory (GBT): state-of-the-art report, Steel Construction. (2013) 6(2):117-131.

[14] Gonçalves R., Camotim D. GBT local and global buckling analysis of aluminium and stainless steel columns, Computers \& Structures. (2004) 82(17-19):1473-1484.

[15] Gonçalves R., Le Grognec P., Camotim D. GBT-based semi-analytical solutions for the plastic bifurcation of thin-walled members, International Journal of Solids and Structures. (2010) 47(1):34-50.

[16] Gonçalves R., Camotim D. Generalized Beam Theory-based finite elements for elastoplastic thin-walled metal members, Thin-Walled Structures. (2011) 49(10):12371245.

[17] Henriques D., Gonçalves R., Camotim D. A physically non-linear GBT-based finite element for steel and steel-concrete beams including shear lag effects, Thin-Walled Structures. (2015) 90:202-215.

[18] Henriques D., Gonçalves R., Camotim D. GBT-based finite element to assess the buckling behaviour of steel-concrete composite beams, Thin-Walled Structures. (2016) 107:207- 
220.

[19] Henriques D., Gonçalves R., Sousa C., Camotim D. GBT-based time-dependent analysis of steel-concrete composite beams including shear lag and concrete cracking effects, ThinWalled Structures. (2020) 150:106706.

[20] Gonçalves R., Camotim D. Geometrically non-linear Generalized Beam Theory for elastoplastic thin-walled metal members, Thin-Walled Structures. (2012) 51:121-129.

[21] Simo J., Taylor R. Consistent tangent operators for rate-independent elastoplasticity, Computer Methods in Applied Mechanics and Engineering. (1985) 48(1):101-118.

[22] MATLAB, version 7.10.0 (R2010a), The MathWorks Inc., Massachusetts, (2010).

[23] Manta D., Gonçalves R., Camotim D. Combining shell and GBT-based finite elements: linear and bifurcation analysis, Thin-Walled Structures. (2020) 152:106665.

[24] Manta D., Gonçalves R., Camotim D. Combining shell and GBT-based finite elements: plastic analysis with adaptive mesh refinement, Thin-Walled Structures. (2021) 158:107205.

[25] Bathe K.J. Finite Element Procedures ( $1^{\text {st }}$ ed.), Prentice-Hall Inc., New Jersey, USA, (1996).

[26] Bathe K.J., Dvorkin E.N. A four-node plate bending element based on Mindlin/Reissner plate theory and a mixed interpolation, International Journal for Numerical Methods in Engineering. (1985) 21(2):367-383.

[27] Ritto-Corrêa M., Camotim D. Integration algorithm for J2 elastoplasticity under arbitrary mixed stress-strain control, International Journal for Numerical Methods in Engineering. (2001) 50(5):1213-1232.

[28] Gonçalves R., Bebiano R., Camotim D. On the shear deformation modes in the framework of Generalised Beam Theory, Thin-Walled Structures. (2014) 84:325-334.

[29] Bebiano R., Gonçalves R., Camotim D. A cross-section analysis procedure to rationalise and automate the performance of GBT-based structural analyses, Thin-Walled Structures. (2015) 92:29-47.

[30] Bebiano R., Camotim D., Gonçalves R. GBTUL 2.0 - a second-generation code for the GBT-based buckling and vibration analysis of thin-walled members, Thin-Walled Structures. (2018) 124:235-257.

[31] Hitziger T., Mackens W., Voss H. A condensation-projection method for generalized eigenvalue problems, High Performance Computing in Engineering, vol. 1, H. Power and C.A. Brebbia (eds.), Computational Mechanics Publications, Southampton, 239-282, (1995). 